Louisiana State University

LSU Digital Commons

Faculty Publications

Department of Mathematics

2-8-2015

\title{
Stability analysis for systems with time-varying delay: Trajectory based approach
}

Frederic Mazenc

Laboratoire des Signaux et Systèmes

Michael Malisoff

Louisiana State University

Silviu Iulian Niculescu

Laboratoire des Signaux et Systèmes

Follow this and additional works at: https://digitalcommons.Isu.edu/mathematics_pubs

\section{Recommended Citation}

Mazenc, F., Malisoff, M., \& Niculescu, S. (2015). Stability analysis for systems with time-varying delay: Trajectory based approach. Proceedings of the IEEE Conference on Decision and Control, 54rd IEEE Conference on Decision and Control,CDC 2015, 1811-1816. https://doi.org/10.1109/CDC.2015.7402473

This Conference Proceeding is brought to you for free and open access by the Department of Mathematics at LSU Digital Commons. It has been accepted for inclusion in Faculty Publications by an authorized administrator of LSU Digital Commons. For more information, please contact ir@lsu.edu. 


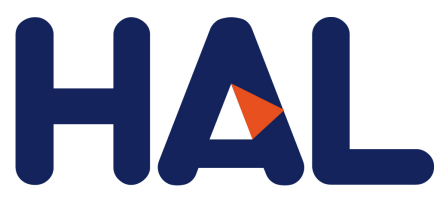

archives-ouvertes

\section{Stability Analysis for Systems with Time-Varying Delay: Trajectory Based Approach}

Frederic Mazenc, Michael Malisoff, Silviu-Iulian Niculescu

\section{To cite this version:}

Frederic Mazenc, Michael Malisoff, Silviu-Iulian Niculescu. Stability Analysis for Systems with TimeVarying Delay: Trajectory Based Approach. 54th IEEE Conference on Decision and Control (CDC), Dec 2015, Ozaka, Japan. 10.1109/cdc.2015.7402473 . hal-01248070

\section{HAL Id: hal-01248070 \\ https://hal.inria.fr/hal-01248070}

Submitted on 23 Dec 2015

HAL is a multi-disciplinary open access archive for the deposit and dissemination of scientific research documents, whether they are published or not. The documents may come from teaching and research institutions in France or abroad, or from public or private research centers.
L'archive ouverte pluridisciplinaire HAL, est destinée au dépôt et à la diffusion de documents scientifiques de niveau recherche, publiés ou non, émanant des établissements d'enseignement et de recherche français ou étrangers, des laboratoires publics ou privés. 


\section{Stability Analysis for Systems with Time-Varying Delay: Trajectory Based Approach}

Frederic Mazenc
Michael Malisoff
Silviu-Iulian Niculescu

\begin{abstract}
Recent work by Mazenc and Malisoff provided a trajectory-based approach to proving stability of time-varying systems with time-varying delay. It uses a contraction lemma, instead of Lyapunov-Krasovskii or Razumikhin functions. Here, we use their lemma, and a Lyapunov function for an undelayed system, to provide a new method to prove stability of linear continuous-time time-varying systems with time-varying bounded delays. No constraint on the upper bound of delay is imposed, nor do we need any differentiability of the delay. Instead, we use an upper bound on an integral average of the delay. We prove input-to-state stability under disturbances.
\end{abstract}

Key Words: delay, time-varying, stability

\section{INTRODUCTION}

Delays occur in many control systems, e.g., where the current state is not available for measurement. Stability analysis for systems with time-varying delays is of theoretical and applied significance, especially when the delays have discontinuities. Motivations arise from networked systems, and systems with nonconstant delays have been studied; see [1], [4], [8], [10, Chapt. 6], [15], [16], [23], and [25]. Such systems can be challenging, because standard frequency domain and Lyapunov-Krasovskii techniques do not apply.

Time-varying delays $h(t)$ model many phenomena, such as delayed muscle response due to fatigue [5] (in which case $h$ may increase), and sampling in controllers [7] (where $h^{\prime}(t)=1$ almost everywhere). Also, the upper bound on the time-varying delays that preserve stability may be larger than the corresponding bound for constant delays; see [17] and the discussion [18] of quenching. On the other hand, even if a system $x^{\prime}(t)=\mathcal{F}(x(t), x(t-\tau))$ is exponentially stable for all constant delays $\tau$ in a given interval $\left[0, \tau_{\max }\right]$, then it may not necessarily be the case that $x^{\prime}(t)=\mathcal{F}(x(t), x(t-$ $h(t)))$ is exponentially stable for all nonconstant delays $h:[0, \infty) \rightarrow\left[0, \tau_{\max }\right]$; see the Markus-Yanabe instability phenomenon [11], [13], [19]. Moreover, there may be different delays occurring in different components of the state entering the control. This motivates developing stability results for cases where the delay is not necessarily constant.

The study of systems with time-varying delays is even more difficult when, in addition, the systems being studied

Mazenc and Niculescu are with EPI DISCO INRIA-Saclay, Laboratoire des Signaux et Systèmes (L2S, UMR CNRS 8506), CNRS, CentraleSupélec, Université Paris-Sud, 3 rue Joliot Curie, 91192, Gifsur-Yvette, France. frederic.mazencel2s. centralesupelec.fr and NICULESCUQIss . supelec. fr. Mazenc was supported by l'Institut pour le Contrôle et la Décision de l'Idex Paris-Saclay (iCODE)

Malisoff is with the Department of Mathematics, 303 Lockett Hall, Louisiana State University (LSU), Baton Rouge, LA 70803-4918, USA. malisoff@lsu.edu. Supported by NSF-ECCS Grants 1102348 and 1408295 and Roy P. Daniels Professorship \#3 in LSU College of Science. are time-varying when the delay is zero [10]. The motivation for studying time-varying control systems is strong, since tracking problems for nonlinear control systems generally lead to time-varying systems with time-varying linear approximations [22]. Many contributions require that the largest value of the delay be sufficiently small, or that the matrices of the vector field belong to special compact sets [10, Chapt. 6]. Another classical assumption is that the delay $h(t)$ is such that the function $\phi(t)=t-h(t)$ is invertible [16]. The contribution [4] is an exception. In [4], the function $\phi(t)=t-h(t)$ is not assumed to be invertible; instead, an averaging assumption on an integral of $\left|h^{\prime}\right|^{2}$ is imposed.

The present paper owes a great deal to [4], because we also use an assumption on an integral involving $h$. However, we do not require differentiability of $h$, and [4] is restricted to constant coefficient linear systems with predictive controls. We establish our results by applying the trajectory-based approach from [20]. The approach from [20] has the potential advantages that (i) it does not require the Lyapunov or small gain conditions that are prevalent in the literature and (ii) it applies to many classes of time-varying systems with timevarying delay. We are not aware of any other technique that can establish the results that we provide in the present paper.

The restrictions on the size of the delay in [9] and [21] are not in general satisfied by the systems we consider here. Moreover, we do not require the time-varying matrices involving the vector fields to belong to specific compact sets, as was imposed in [10, Chapt. 6]. Unlike [4], we consider linear time-varying systems with a time-varying piecewise continuous delay $h(t)$. The requirements from [16] may be violated by our systems, and our results ensure exponential stability in cases where the existing Razumikhin and Lyapunov-Krasovskii methods do not seem to apply.

In Section II, we explain our notation and our key lemma from [20] that we use to prove our main results in Sections III-IV. Section V has illustrating examples. In Section VI, we summarize our findings and suggest future research topics. Sections III-V consist of previously unpublished, original results which are not being submitted elsewhere.

\section{NOTATION AND KEY LEMMA FROM [20]}

In what follows, all dimensions are arbitrary. The usual Euclidean norm of vectors, and the induced norm of matrices, are denoted by $|\cdot|$. For any measurable essentially bounded $\mathbb{R}^{n}$ valued function $\phi$ having an interval $\mathcal{I}$ in its domain, let $|\phi|_{\mathcal{I}}$ be its essential supremum over $\mathcal{I}$. Let $C^{1}$ denote the set of all continuously differentiable functions, where the domains and ranges will be clear from the context. Given 
any constant $\tau>0$, we let $C\left([-\tau, 0], \mathbb{R}^{n}\right)$ denote the set of all continuous $\mathbb{R}^{n}$-valued functions that are defined on $[-\tau, 0]$. We abbreviate this set as $C_{\text {in }}$, and call it the set of all initial functions. By input-to-state stability (or ISS) of a system of the form $\dot{x}(t)=f\left(t, x_{t}, u\right)$ with respect to functions $u:[0, \infty) \rightarrow \mathcal{D}$ that are valued in some set $\mathcal{D}$, we mean that there are $\beta \in \mathcal{K} \mathcal{L}$ and $\gamma \in \mathcal{K}_{\infty}$ such that for all initial functions $x_{0}$ and all locally bounded piecewise continuous functions $u:[0, \infty) \rightarrow \mathcal{D}$, the corresponding unique solution $x(t)$ of the system with initial function $x_{0}$ satisfies $|x(t)| \leq \beta\left(\left|x_{0}\right|_{[-\tau, 0]}, t\right)+\gamma\left(|u|_{[0, t]}\right)$ for all $t \geq 0$, where $\mathcal{K} \mathcal{L}$ and $\mathcal{K}_{\infty}$ are the standard classes of comparison functions [14] and for simplicity we always take the initial times to be 0 . By piecewise continuous (resp., $C^{1}$ ), we mean continuous (resp., $C^{1}$ ) except at finitely many points on each bounded interval; this includes the classes of all continuous and $C^{1}$ functions when the finite sets are empty. Also, for any continuous function $\varphi:[-\tau, \infty) \rightarrow \mathbb{R}^{n}$ and all $t \geq 0$, we define $\varphi_{t}$ by $\varphi_{t}(m)=\varphi(t+m)$ for all $m \in[-\tau, 0]$, i.e., $\varphi_{t} \in C_{\text {in }}$ is the translation operator. The special case of ISS where $u$ is zero and $\beta(s, t)=\bar{c}_{1} s e^{-\bar{c}_{2} t}$ for some constants $\bar{c}_{1}>0$ and $\bar{c}_{2}>0$ is global exponential stability (GES). We use this trajectory-based stability result from [20]:

Lemma 1: Let $T^{*}>0$ be a constant. Let a function $w$ : $\left[-T^{*}, \infty\right) \rightarrow[0, \infty)$ admit a sequence $\left\{v_{i}\right\}$ and positive constants $\bar{v}_{a}$ and $\bar{v}_{b}$ such that $v_{0}=0, v_{i+i}-v_{i} \in\left[\bar{v}_{a}, \bar{v}_{b}\right]$ for all $i \geq 0, w$ is continuous on $\left[v_{i}, v_{i+1}\right)$ for all $i \geq 0$, and the left $\operatorname{limit}_{\lim _{v \rightarrow v_{i}-}} w(v)$ exists and is finite for each $i \in \mathbb{N}$. Let $d:[0, \infty) \rightarrow[0, \infty)$ be any piecewise continuous function. Assume that there is a constant $\rho \in(0,1)$ such that

$$
w(t) \leq \rho|w|_{\left[t-T^{*}, t\right]}+d(t)
$$

holds for all $t \geq 0$. Then

$$
w(t) \leq|w|_{\left[-T^{*}, 0\right]} e^{\frac{\ln (\rho)}{T^{*}} t}+\frac{1}{(1-\rho)^{2}}|d|_{[0, t]}
$$

holds for all $t \geq 0$.

See Section IV-C for more comparisons with [20].

\section{Preliminary Result}

\section{A. Statement and Proof of Result}

We first study systems

$$
\dot{x}(t)=A(t) x(t)+B(t) x(t-h(t))+u(t)
$$

whose stabilizing part $A(t) x(t)$ consists of a term without delay (but see the next section below for other cases). In (3), we assume that the state $x$ is valued in $\mathbb{R}^{n}$, the initial conditions are in $C_{\text {in }}, h:[0, \infty) \rightarrow[0, \infty)$ is a piecewise continuous function, and $A:[0, \infty) \rightarrow \mathbb{R}^{n \times n}$, $B:[0, \infty) \rightarrow \mathbb{R}^{n \times n}$, and $u:[0, \infty) \rightarrow \mathbb{R}^{n}$ are piecewise continuous functions that are bounded in norm over $[0, \infty)$. The functions $u$ represent uncertainty.

We show that even when the term $B(t) x(t-h(t))$ does not help stabilize the system, we can establish robust stability in cases where the classical stability analysis techniques do not seem to apply; see our example in Section V-A. We introduce

$$
\dot{z}(t)=A(t) z(t)
$$

with $z$ valued in $\mathbb{R}^{n}$ and the following two assumptions:

Assumption 1: There exist a continuous function $p_{1}$ : $[0, \infty) \rightarrow \mathbb{R}$, a $C^{1}$ function $P:[0, \infty) \rightarrow \mathbb{R}^{n \times n}$ such that $P(t)$ is symmetric and positive definite for all $t \geq 0$, and positive constants $p_{2}, p_{3}$, and $p_{4}$ such that

$$
\left|p_{1}(t)\right| \leq p_{4}
$$

and

$$
p_{2}|z|^{2} \leq V(t, z) \leq p_{3}|z|^{2}
$$

hold for all $t \geq 0$ and $z \in \mathbb{R}^{n}$, where

$$
V(t, z)=z^{\top} P(t) z
$$

and such that along all trajectories of (4), we have

$$
\dot{V}(t, z) \leq-p_{1}(t) V(t, z)
$$

for all $t \geq 0$.

Assumption 2: There is a constant $h_{*} \geq 0$ such that

$$
0 \leq h(t) \leq h_{*} \text { for all } t \geq 0 .
$$

Also, there are constants $T>0$ and $\delta \in(0,1)$ such that

$$
\begin{aligned}
& \int_{t-T}^{t} \frac{2}{p_{2}}\left(|P(m) B(m)|+p_{3}|u(m)|\right) e^{-\int_{m}^{t} p_{1}(\ell) \mathrm{d} \ell} \mathrm{d} m \\
& \quad+e^{-\int_{t-T}^{t} p_{1}(m) \mathrm{d} m} \leq \delta \text { holds for all } t \geq T,
\end{aligned}
$$

where $P$ and the $p_{i}$ 's are from Assumption 1 .

Our first result for (3) is as follows (but see Remark 1 for a way to convert the conclusion of the following theorem into an ISS estimate with respect to $u$ that holds for all $t \geq 0$ ):

Theorem 1: If Assumptions 1-2 hold, then all solutions $x(t)$ of (3) satisfy

$$
\begin{aligned}
|x(t)| \leq & \sqrt{\frac{p_{3}}{p_{2}} e^{\frac{\ln (\delta)}{T+h_{*}}\left(t-T-h_{*}\right)}}|x|_{\left[0, T+h_{*}\right]} \\
& +\sqrt{\frac{2 T p_{3} e^{T p_{4}}|u|_{\left[h_{*}, t\right]}}{p_{2}(1-\delta)^{2}}}
\end{aligned}
$$

for all $t \geq T+h_{*}$.

Proof: From Assumption 1, it follows that the time derivative of $V$ along all trajectories of (3) satisfies

$$
\begin{aligned}
\dot{V}(t) \leq & -p_{1}(t) V(t, x(t))+2 x(t)^{\top} P(t) u(t) \\
& +2 x(t)^{\top} P(t) B(t) x(t-h(t))
\end{aligned}
$$

for all $t \geq 0$. Using (6) and considering the possibilities $\sqrt{V(t, x(t)) / p_{2}} \leq 1$ and $\sqrt{V(t, x(t)) / p_{2}}>1$, we get

$$
\begin{aligned}
\dot{V}(t) \leq & -p_{1}(t) V(t, x(t))+2|P(t)||x(t)||u(t)| \\
+ & 2|P(t) B(t)||x(t)||x(t-h(t))| \\
\leq \quad & p_{1}(t) V(t, x(t))+2|P(t) B(t)| \\
& \times \sqrt{\frac{V(t, x(t))}{p_{2}}} \sqrt{\frac{V(t-h(t), x(t-h(t))}{p_{2}}} \\
+ & 2 p_{3} \sqrt{\frac{V(t, x(t))}{p_{2}}}|u(t)|
\end{aligned}
$$

and therefore also

$$
\begin{aligned}
\dot{V}(t) \leq & -p_{1}(t) V(t, x(t)) \\
& +\frac{2|P(t) B(t)|}{p_{2}} \sup _{s \in\left[t-h_{*}, t\right]} V(s, x(s)) \\
& +2 p_{3}\left[\frac{1}{p_{2}}|u(t)| V(t, x(t))+|u(t)|\right]
\end{aligned}
$$


for all $t \geq h_{*}$, where we also used the fact that $|P(t)| \leq$ $p_{3}$ holds for all $t \geq 0$, which follows because (6) gives $|\sqrt{P(t)} z| \leq \sqrt{p_{3}}|z|$ and therefore also

$$
|P(t) z|=|\sqrt{P(t)}(\sqrt{P(t)} z)| \leq p_{3}|z|
$$

for all $z \in \mathbb{R}^{n}$ and $t \geq 0$. Consequently,

$$
\begin{aligned}
& \dot{V}(t) \leq-p_{1}(t) V(t, x(t))+2 p_{3}|u(t)| \\
& +\frac{2\left(|P(t) B(t)|+p_{3}|u(t)|\right)}{p_{2}} \sup _{s \in\left[t-h_{*}, t\right]} V(s, x(s))
\end{aligned}
$$

for all $t \geq h_{*}$.

Lemma A.1 in the appendix below applied with $a(t)=$ $p_{1}(t), a_{s}=p_{4}, \lambda(t)=2 p_{3}|u(t)|, g=h_{*}$ and $X(t)=$ $V(t, x(t))$ and Assumption 2 allow us to conclude that

$$
\begin{aligned}
V(t, x(t)) \leq & \sup _{s \in\left[0, T+h_{*}\right]} V(s, x(s)) e^{\frac{\ln (\delta)}{T+h_{*}}\left(t-T-h_{*}\right)} \\
& +\frac{2 T p_{3} e^{T p_{4}}|u|_{\left[h_{*}, t\right]}}{(1-\delta)^{2}}
\end{aligned}
$$

holds for all $t \geq h_{*}+T$. From (6), it follows that

$$
\begin{aligned}
p_{2}|x(t)|^{2} \leq & p_{3}|x|_{\left[0, T+h_{*}\right]}^{2} e^{\frac{\ln (\delta)}{T+h_{*}}\left(t-T-h_{*}\right)} \\
& +\frac{2 T p_{3} e^{T p_{4}}|u|_{\left[h_{*}, t\right]}}{(1-\delta)^{2}}
\end{aligned}
$$

for all $t \geq h_{*}+T$. This allows us to conclude.

Remark 1: Our conclusion (11) differs from an ISS estimate because it only holds for $t \geq T+h_{*}$, and the usual procedure for using an integrating factor to extend the ISS estimate to $[0, \infty)$ does not apply, because of the time delay. However, we can use the following simple Gronwall inequality argument to extend the ISS estimate (11) to all of $[0, \infty)$. Set $\bar{A}=\max \left\{\sup _{t \in\left[0, T+h_{*}\right]}|A(t)|, \sup _{t \in\left[0, T+h_{*}\right]}|B(t)|\right\}$. Then the dynamics (3) gives $|\dot{x}(t)| \leq 2 \bar{A}|x|_{\left[t-h_{*}, t\right]}+$ $|u|_{\left[0, T+h_{*}\right]}$ (for all $t \in\left[0, T+h_{*}\right]$ where the corresponding solution is defined). Applying the Fundamental Theorem of Calculus to $x(s)$ on $[0, t]$ for any such $t \in\left[0, T+h_{*}\right]$, and then suping on the left side over $[0, t]$, we get

$$
\begin{aligned}
|x|_{\left[t-h_{*}, t\right] \leq} \leq & |x|_{\left[-h_{*}, 0\right]}+2 \bar{A} \int_{0}^{t}|x|_{\left[s-h_{*}, s\right]} \mathrm{d} s \\
& +\left(T+h_{*}\right)|u|_{\left[0, T+h_{*}\right]} .
\end{aligned}
$$

Next we apply Gronwall's inequality to $\phi(s)=|x|_{\left[s-h_{*}, s\right]}$ on $[0, t]$ and use the fact that trajectory values on $[0, t]$ do not depend on future values of $u$ (i.e., causality) to get

$$
\begin{aligned}
& |x|_{\left[t-h_{*}, t\right]} \\
& \leq\left(|x|_{\left[-h_{*}, 0\right]} e^{T+h_{*}-t}+\left(T+h_{*}\right)|u|_{[0, t]}\right) e^{2 \bar{A}\left(T+h_{*}\right)}
\end{aligned}
$$

for all $t \in\left[0, T+h_{*}\right]$ and therefore also

$$
\begin{aligned}
|x(t)| \leq & e^{2 \bar{A}\left(T+h_{*}\right)}|x|_{\left[-h_{*}, 0\right]} e^{T+h_{*}-t} \\
& +\left(T+h_{*}\right) e^{2 \bar{A}\left(T+h_{*}\right)}|u|_{[0, t]}
\end{aligned}
$$

for all $t \in\left[0, T+h_{*}\right]$. Substituting (21) into (11) gives

$$
\begin{aligned}
|x(t)| \leq & \sqrt{\frac{p_{3}}{p_{2}} e^{\frac{\ln (\delta)}{T+h_{*}}\left(t-T-h_{*}\right)}} e^{(2 \bar{A}+1)\left(T+h_{*}\right)}|x|_{\left[-h_{*}, 0\right]} \\
& +\sqrt{p_{3} / p_{2}}\left(T+h_{*}\right) e^{2 \bar{A}\left(T+h_{*}\right)}|u|_{[0, t]} \\
& +\sqrt{\frac{2 T p_{3} e^{T p_{4}}|u|_{[0, t]}}{p_{2}(1-\delta)^{2}}}
\end{aligned}
$$

for all $t \geq T+h_{*}$. The final ISS estimate is obtained by adding (21) and (22) and then dividing both sides of the result by 2 to get an ISS estimate that holds for all $t \geq 0$.

\section{B. Notable Features of Theorem 1}

Before turning to our main result of this paper, we discuss several features of Theorem 1 . We do not require $p_{1}$ to be nonnegative valued, but Assumptions 1-2 imply that (4) is exponentially stable to zero (GES), by taking $B=0$. Since we do not require $p_{1}$ to be periodic, it is unclear whether $V$ can be transformed into a strict Lyapunov function for (4).

Theorem 1 leads to a bound on $B$ such that (3) is GES if (4) is GES. Moreover, we can find a bound on $B$ that is independent of the bound $h_{*}$ on $h(t)$. To see how this can be done, assume that (4) is GES and that $A$ is bounded and continuous. For simplicity, we assume that the perturbation $u$ in (3) is the zero function. Then standard results (e.g., [14, Theorem 4.14]) allow us to satisfy Assumption 1 with a constant positive value of $p_{1}(t)=\bar{p}_{1}$. Then (10) holds if there are constants $T>0$ and $\delta \in(0,1)$ such that

$$
e^{-T \bar{p}_{1}}+\frac{2 p_{3}|B|_{[0, \infty)}}{p_{2}} \int_{0}^{T} e^{-\bar{p}_{1} m} \mathrm{~d} m<\delta,
$$

or equivalently, if $|B|_{[0, \infty)}<\bar{p}_{1} p_{2} /\left(2 p_{3}\right)$ is satisfied.

Here is an analog where $p_{1}$ takes both positive and negative values. Assume that we are given a GES system

$$
\dot{z}(t)=\mathcal{N} z(t)
$$

for some constant matrix $\mathcal{N}$, and any bounded continuous function $q: \mathbb{R} \rightarrow \mathbb{R}$ that admits constants $q_{0}>0$ and $T>0$ such that for all $t \in \mathbb{R}$, we have $\int_{t-T}^{t} q(\ell) \mathrm{d} \ell \geq q_{0}$. For instance, $q$ can be a persistency of excitation function like $q(\ell)=\sin ^{2}(\ell)$. Then solving a Riccati equation provides a positive definite symmetric matrix $P$ and positive constants $\underline{\alpha}, \bar{\alpha}$, and $\alpha_{*}$ such that the time derivative of $V(z)=z^{\top} P z$ along all trajectories of (24) satisfies $\dot{V}(z)=z^{\top}\left(\mathcal{N}^{\top} P+\right.$ $P \mathcal{N}) z \leq-\alpha_{*} V(z)$ and such that $\underline{\alpha}|z|^{2} \leq V(z) \leq \bar{\alpha}|z|^{2}$ for all $t \geq 0$ and $z \in \mathbb{R}^{n}$. Set $A(t)=q(t) \mathcal{N}$. Then Assumption 1 holds with the choices $p_{1}(t)=\alpha_{*} q(t), p_{2}=\underline{\alpha}, p_{3}=\bar{\alpha}$, and $p_{4}=\left|p_{1}\right|_{[0, \infty)}$. Then we can argue as in the preceding paragraph to conclude that

$$
\dot{x}(t)=q(t) \mathcal{N} x(t)+B(t) x(t-h(t))
$$

is GES for all piecewise continuous functions $h:[0, \infty) \rightarrow$ $[0, \infty)$, and for all choices of $B: \mathbb{R} \rightarrow \mathbb{R}^{n \times n}$ such that

$$
\begin{aligned}
& \sup _{t \geq T}\left\{\exp \left(-\alpha_{*} \int_{t-T}^{t} q(\ell) \mathrm{d} \ell\right)\right. \\
& \left.+\int_{t-T}^{t} \frac{2 p_{3}}{p_{2}}|B(m)| \exp \left(-\alpha_{*} \int_{m}^{t} q(\ell) \mathrm{d} \ell\right) \mathrm{d} m\right\}<1,
\end{aligned}
$$

which is satisfied if

$$
\begin{aligned}
& |B|_{[0, \infty)}< \\
& \inf _{t \geq 0} \frac{p_{2}}{2 p_{3}}\left(\int_{t-T}^{t} \exp \left(-\alpha_{*} \int_{m}^{t} q(\ell) \mathrm{d} \ell\right) \mathrm{d} m\right)^{-1}\left(1-e^{-\alpha_{*} q_{0}}\right)
\end{aligned}
$$

holds.

Similar reasoning applies if $\mathcal{N}$ is time varying, as long as $\dot{z}=\mathcal{N}(t) z$ admits a time invariant Lyapunov function of the form $V(z)=z^{\top} P z$. In summary, Theorem 1 can be 
viewed as a robustness result for GES of the stable system (4) with respect to the potentially destabilizing added terms $B(t) x(t-h(t))+u(t)$ with time-varying delays $h(t)$.

\section{MAIN Result}

\section{A. Statement and Proof of Main Result}

We consider systems (3) in situations where (4) is not necessarily exponentially stable. For the sake of simplicity, we do not the consider the case where an additive disturbance $u$ is present (but additive disturbances can also be handled, by a variant of the arguments used in the previous section). Let us introduce the system

$$
\dot{z}(t)=H(t) z(t)
$$

where $H(t)=A(t)+B(t)$, and the following assumptions:

Assumption 3: The matrices $A$ and $B$ and the delay $h$ are piecewise continuous. Also, there exist a bounded piecewise continuous function $p_{1}:[0, \infty) \rightarrow \mathbb{R}$, positive constants $p_{2}$ and $p_{3}$, and a $C^{1}$ function $P:[0, \infty) \rightarrow \mathbb{R}^{n \times n}$ such that $P(t)$ is symmetric and positive definite for all $t \geq 0$, for which: For all $t \geq 0$ and $z \in \mathbb{R}^{n}$, we have

$$
p_{2}|z|^{2} \leq V(t, z) \leq p_{3}|z|^{2},
$$

where $V(t, z)=z^{\top} P(t) z$ and the time derivative of $V$ along all trajectories of (26) satisfies

$$
\dot{V}(t, z) \leq-p_{1}(t) V(t, z)
$$

for all $t \geq 0$.

Assumption 4: There is a constant $h_{*} \geq 0$ such that

$$
0 \leq h(t) \leq h_{*}
$$

hold for all $t \geq 0$. Also, there exist constants $T>0$ and $\delta \in(0,1)$ such that with the choice

$$
r(t)=\frac{2|P(t) B(t)|}{p_{2}} \int_{t-h(t)}^{t}[|A(m)|+|B(m)|] \mathrm{d} m,
$$

we have

$$
e^{-\int_{t-T}^{t} p_{1}(m) \mathrm{d} m}+\int_{t-T}^{t} r(m) e^{-\int_{m}^{t} p_{1}(\ell) \mathrm{d} \ell} \mathrm{d} m \leq \delta
$$

for all $t \geq 2\left(T+h_{*}\right)$. Also, $A$ and $B$ are bounded.

We prove:

Theorem 2: If the system (3) satisfies Assumptions 3-4, then (3) satisfies GES.

Proof: For all $t \geq h_{*}$, we have

$$
\begin{aligned}
\dot{x}(t) & =H(t) x(t)-B(t)[x(t)-x(t-h(t))] \\
& =H(t) x(t)-B(t) \int_{t-h(t)}^{t} \dot{x}(m) \mathrm{d} m
\end{aligned}
$$

Using the expression for $\dot{x}(m)$, we obtain $\dot{x}(t)=H(t) x(t)-$ $B(t) \mathcal{L}(t)$ for all $t \geq 2 h_{*}$, where

$\mathcal{L}(t)=\int_{t-h(t)}^{t}[A(m) x(m)+B(m) x(m-h(m))] \mathrm{d} m$.

From Assumption 3, we deduce that for all $t \geq 2 h_{*}$, we have

$$
\dot{V}(t) \leq-p_{1}(t) V(t, x(t))-2 x(t)^{\top} P(t) B(t) \mathcal{L}(t) .
$$

As an immediate consequence, we get

$$
\begin{aligned}
\dot{V}(t) \leq & -p_{1}(t) V(t, x(t)) \\
& +2|P(t) B(t)||x(t)||\mathcal{L}(t)| \\
\leq & -p_{1}(t) V(t, x(t)) \\
& +2|P(t) B(t)|\left|\sqrt{V_{c} / p_{2}}\right|_{\left[t-2 h_{*}, t\right]} \mathrm{d} m \\
& \times \int_{t-h(t)}^{t} A^{\sharp}(m)\left|\sqrt{V_{c} / p_{2}}\right|_{\left[t-2 h_{*}, t\right]}, \\
= & -p_{1}(t) V(t, x(t))+r(t)\left|V_{c}\right|_{\left[t-2 h_{*}, t\right]},
\end{aligned}
$$

where $V_{c}(m)=V(m, x(m))$ and

$$
A^{\sharp}(m)=|A(m)|+|B(m)| .
$$

Lemma A.1 in the appendix below and Assumption 4 allow us to conclude that $V(t, x(t))$ converges exponentially to zero. Then (27) implies that $x(t)$ converges exponentially to the origin. This proves Theorem 2 .

\section{B. Notable Features of Theorem 2}

Remark 2: Since $A$ and $B$ are piecewise continuous and bounded, all solutions of (3) are well-defined and defined over $[0, \infty)$ and the function $r$ in (30) is well-defined.

Remark 3: We do not require $p_{1}$ to be nonnegative valued. Assumptions 3-4 imply that the system (26) is globally exponentially stable.

Remark 4: Theorem 2 is not covered by the results of [10, Chapt. 6]. Also, Theorem 2 can be extended to systems with several time-varying delays. Through Assumption 3, Theorem 2 takes into account the case where the term $B(t) x(t-h(t))$ has a stabilizing effect.

Remark 5: To motivate Assumption 4, consider the case where $p_{1}, P, A$ and $B$ are all constant, and where $p_{1}$ is nonnegative. Then (31) simplifies to

$$
e^{-T p_{1}}+\frac{2|P B|(|A|+|B|)}{p_{2}} \int_{t-T}^{t} h(m) e^{(m-t) p_{1}} \mathrm{~d} m \leq \delta .
$$

This inequality is satisfied if

$$
\frac{1}{T} \int_{t-T}^{t} h(m) \mathrm{d} m \leq \frac{p_{2}}{2|P B|(|A|+|B|)} \frac{\delta-e^{-T p_{1}}}{T}
$$

is satisfied, which pertains to an average value of $h$.

\section{More Comparisons with [20]}

The main strategy in [20] is to prove ISS of systems of the form

$$
\dot{x}(t)=f(t, x(t), \zeta(t, \tau), \Delta(t))
$$

with respect to disturbances $\Delta$, where $\zeta(t, \tau)=\left(x_{1}(t-\right.$ $\left.\left.\tau_{1}(t)\right), x_{2}\left(t-\tau_{2}(t)\right), \ldots, x_{n}\left(t-\tau_{n}(t)\right)\right)$ for piecewise continuous delay functions $\tau_{i}$, by assuming ISS of the system

$$
\dot{x}(t)=f(t, x(t), u(t))
$$

with respect to the combined disturbance $u(t)=$ $(\xi(t, \tau), \Delta(t))$. While our key Lemma 1 is from [20], neither theorem in the present work is covered by [20], e.g., because Assumption 1 does not imply ISS of $\dot{x}(t)=A(t) x(t)+$ $u(t)$, and because the function $p_{1}$ in Assumption 1 is not necessarily positive valued. 


\section{ILLUSTRATIONS}

\section{A. Illustration of Theorem 1}

Let $d>0$ and $c \in(0,1)$ be any constants such that

$$
(1-c) d<\frac{1-e^{-1}}{2} \text {. }
$$

Let $b$ be the periodic function of period 1 defined by

(i) $b(t)=0$ when $t \in[0, c)$ and

(ii) $b(t)=d$ when $t \in[c, 1]$.

Consider the system

$$
\dot{x}(t)=-x(t)+b(t) x(t-1),
$$

where $x$ is valued in $\mathbb{R}$. When $d>1$, Razumikhin's theorem does not apply [10, Chapt. 6]. Moreover, to the best of our knowledge, no Lyapunov-Krasovskii functional construction technique applies to this system.

We check that (41) satisfies Assumptions 1-2 with $V(z)=$ $\frac{1}{2} z^{2}, u \equiv 0, B(t)=b(t)$, and $h(t)=1$. With the notation of Section III, we pick $p_{1}(t)=1$ for all $t \geq 0$ and $P=p_{2}=$ $p_{3}=\frac{1}{2}$. Then

$$
\begin{aligned}
\Xi(t)= & e^{-\int_{t-T}^{t} p_{1}(m) \mathrm{d} m} \\
& +\int_{t-T}^{t} \frac{2|P(m) B(m)|}{p_{2}} e^{-\int_{m}^{t} p_{1}(\ell) \mathrm{d} \ell} \mathrm{d} m
\end{aligned}
$$

satisfies

$$
\Xi(t)=e^{-T}+2 \int_{t-T}^{t} b(m) e^{m-t} \mathrm{~d} m
$$

for all $t \geq 0$. Choosing $T=1$, we obtain

$$
\Xi(t) \leq e^{-1}+2(1-c) d<1,
$$

by (40). Theorem 1 allows us to conclude.

\section{B. Illustration of Theorem 2}

Let $l>0$ be a constant, and $k \in \mathbb{N}$ be an odd integer. We consider the system

$$
\dot{x}(t)=-x(t-h(t)),
$$

where $x$ is valued in $\mathbb{R}$, and the time-varying delay

$$
h(t)=\max \left\{0, l \sin ^{k}(t)\right\} .
$$

To the best of our knowledge, Razumikhin's theorem does not apply to (45), and no Lyapunov-Krasovskii functional construction technique in the literature applies to this system.

With the notation of Section IV, we choose $P(t)=1$, $p_{1}(t)=2, p_{2}=p_{3}=1, H=-1, A=0, B=-1$, and

$$
r(t)=2 h(t)=2 \max \left\{0, l \sin ^{k}(t)\right\} .
$$

Then

$$
\begin{aligned}
& e^{-\int_{t-T}^{t} p_{1}(m) \mathrm{d} m}+\int_{t-T}^{t} r(m) e^{-\int_{m}^{t} p_{1}(\ell) \mathrm{d} \ell} \mathrm{d} m \\
& =e^{-2 T}+\int_{t-T}^{t} 2 \max \left\{0, l \sin ^{k}(m)\right\} e^{2(m-t)} \mathrm{d} m .
\end{aligned}
$$

Pick any constant $T>0$. Then, by the Lebesgue Dominated Convergence Theorem, there exists a constant $k>0$ (which is sufficiently large, and which depends on $l$ and $T$ ) such that there is a constant $\delta \in(0,1)$ satisfying (31) for all $t \geq 0$. Thus, Assumptions 3-4 hold. Theorem 2 allows us to conclude that (45) is globally exponentially stable to 0 . Since $l>0$ is arbitrarily large, we have exponential stability without any constraint on $\sup _{m} h(m)$. To our knowledge, such a result cannot be established by any other technique.

\section{COnClusions And Open PRoblems}

Time-varying delays model many important effects, such as increasing latencies in rehabilitation as a muscle becomes fatigued. Also, sampling in controllers can be modeled using piecewise $C^{1}$ time-varying delays $h(t)$ where $h^{\prime}(t)=1$ almost everywhere, resulting in a sawtooth shaped delay function. We developed new stability analysis techniques for piecewise continuous time-varying linear systems with timevarying delays. Our results can be used when stabilizing control laws must be designed under delays. Since we do not require the standard condition $h^{\prime}(t)<1$, our results are quite general. One of our main tools was a trajectorybased approach from [20], whose advantages are that (a) it circumvents the need for Lyapunov-Krasovskii functionals or to check small-gain conditions and (b) it applies to many classes of time-varying systems with time-varying delay, and therefore is a versatile alternative to traditional methods.

The results we presented here are beyond the scope of [20], e.g., because here we allow $\sup _{t} h(t)$ to be arbitrarily large, and because we introduced a useful new integral condition that allows us to establish the exponential stability. We hope to extend the present paper in several directions. We conjecture that our results can be extended to cases that have distributed delays and to nonlinear systems, including applications to networked systems with uncertainty. It would also be interesting to seek generalizations to adaptive control problems, where the objectives include both tracking and identification of unknown model parameters, as well as to cases where there are also state dependent or rapidly varying delays [2], [3], [6] or sampling [24].

\section{APPENDIX}

The following is a time-varying version of Halanay's lemma [12], and was used to prove Theorems 1-2:

Lemma A.1: Let $X:[0, \infty) \rightarrow[0, \infty)$ be a piecewise $C^{1}$ function that admits constants $g \geq 0$ and $a_{s} \geq 0$ and piecewise continuous functions $a:[0, \infty) \rightarrow\left[-a_{s}, \infty\right), b:$ $[0, \infty) \rightarrow[0, \infty)$, and $\lambda:[0, \infty) \rightarrow[0, \infty)$ such that

$$
\dot{X}(t) \leq-a(t) X(t)+b(t) \sup _{s \in[t-g, t]} X(s)+\lambda(t)
$$

holds for all $t \geq g$, and assume that the left limit $\lim _{t \rightarrow p^{-}} X(t)$ exists and is finite at each $p \geq 0$. Assume that there exist two constants $T>0$ and $\delta \in(0,1)$ such that

$$
e^{-\int_{t-T}^{t} a(m) \mathrm{d} m}+\int_{t-T}^{t} b(m) e^{-\int_{m}^{t} a(\ell) \mathrm{d} \ell} \mathrm{d} m \leq \delta
$$

holds for all $t \geq T+g$. Then, the inequality

$$
X(t) \leq|X|_{[0, T+g]} e^{\frac{\ln (\delta)}{T+g}(t-T-g)}+\frac{T e^{T a_{s}}|\lambda|_{[g, t]}}{(1-\delta)^{2}}
$$

holds for all $t \geq T+g$. 
Proof: Let

$$
\zeta(t)=e^{\int_{0}^{t} a(m) \mathrm{d} m} X(t) .
$$

Then, we can use (A.1) to get

$$
\begin{aligned}
\dot{\zeta}(t) & =e^{\int_{0}^{t} a(m) \mathrm{d} m}[\dot{X}(t)+a(t) X(t)] \\
& \leq e^{\int_{0}^{t} a(m) \mathrm{d} m}\left[b(t) \sup _{s \in[t-g, t]} X(s)+\lambda(t)\right]
\end{aligned}
$$

for all $t \geq g$. It follows that for all $t \geq T+g$, we have

$$
\begin{aligned}
& \zeta(t)-\zeta(t-T) \leq \\
& \int_{t-T}^{t} e^{\int_{0}^{m} a(s) \mathrm{d} s}\left[b(m) \sup _{s \in[m-g, m]} X(s)+\lambda(m)\right] \mathrm{d} m .
\end{aligned}
$$

From the definition of $\zeta$, we deduce that

$$
\begin{aligned}
X(t) \leq & e^{-\int_{t-T}^{t} a(m) \mathrm{d} m} X(t-T) \\
& +\int_{t-T}^{t} b(m) e^{-\int_{m}^{t} a(\ell) \mathrm{d} \ell} \sup _{s \in[m-g, m]} X(s) \mathrm{d} m \\
& +\int_{t-T}^{t} \lambda(m) e^{-\int_{m}^{t} a(\ell) \mathrm{d} \ell} \mathrm{d} m \\
\leq & {\left[e^{-\int_{t-T}^{t} a(m) \mathrm{d} m}+\int_{t-T}^{t} b(m) e^{-\int_{m}^{t} a(\ell) \mathrm{d} \ell} \mathrm{d} m\right] } \\
& \times \sup _{s \in[t-T-g, t]} X(s) \\
& +\int_{t-T}^{t} \lambda(m) e^{-\int_{m}^{t} a(\ell) \mathrm{d} \ell} \mathrm{d} m .
\end{aligned}
$$

From (A.2), it follows that, for all $t \geq T+g$, the inequality

$$
X(t) \leq \delta \sup _{s \in[t-T-g, t]} X(s)+\kappa(t),
$$

where

$$
\kappa(t)=e^{T a_{s}} \int_{t-T}^{t} \lambda(m) \mathrm{d} m .
$$

We now apply Lemma 1 with

$$
\begin{aligned}
& T^{*}=T+g, \quad w(t)=X(t+T+g), \\
& \text { and } d(t)=\kappa(t+T+g) .
\end{aligned}
$$

Since (A.7) implies that

$$
w(t) \leq \delta \sup _{s \in[t-T-g, t]} w(s)+\kappa(t+T+g)
$$

for all $t \geq 0$, we deduce from Lemma 1 that

$$
w(t) \leq|w|_{[-T-g, 0]} e^{\frac{\ln (\delta)}{T+g} t}+\frac{1}{(1-\delta)^{2}}|\kappa|_{[T+g, t+T+g]}
$$

for all $t \geq 0$. Hence, for all $t \geq T+g$, the inequality

$$
X(t) \leq|X|_{[0, T+g]} e^{\frac{\ln (\delta)}{T+g}(t-T-g)}+\frac{|\kappa|_{[T+g, t+T+g]}}{(1-\delta)^{2}}
$$

is satisfied for all $t \geq T+g$, which proves the lemma, since $X(t)$ has no dependence on values $\kappa(s)$ for values of $s$ on the interval $[t, t+T+g]$.

\section{REFERENCES}

[1] N. Bekiaris-Liberis and M. Krstic. Nonlinear Control under Nonconstant Delays. Advances in Design and Control, Volume 25. Society for Industrial and Applied Mathematics, Philadelphia, PA, 2013.

[2] N. Bekiaris-Liberis and M. Krstic. Compensation of state-dependent input delay for nonlinear systems. IEEE Transactions on Automatic Control 58(2):275-289, 2013.

[3] N. Bekiaris-Liberis and M. Krstic. Robustness of nonlinear predictor feedback laws to time- and state-dependent delay perturbations. Automatica 49(6):1576-1590, 2013.

[4] D. Bresch-Pietri and N. Petit. Robust compensation of a chattering time-varying input delay. In Proceedings of the IEEE Conference on Decision and Control, Los Angeles, CA, 2014, pp. 457-462.

[5] R. Downey, R. Kamalapurkar, N. Fischer, and W. Dixon. Compensating for fatigue-induced time-varying delayed muscle response in neuromuscular electrical stimulation control. In: Recent Results on Nonlinear Time Delayed Systems, I. Karafyllis, M. Malisoff, F. Mazenc, and P. Pepe, Eds., Advances in Dynamics and Delays Series Volume 4, Springer, New York, 2016, pp. 143-161.

[6] E. Fridman and S.-I. Niculescu. On complete Lyapunov-Krasovskii functional techniques for uncertain systems with fast-varying delays. International Journal of Robust and Nonlinear Control 18(3):364-374, 2008.

[7] E. Fridman, A. Seuret, and J.-P. Richard. Robust sampled-data stabilization of linear systems: An input delay approach. Automatica 40(8):1141-1446, 2004

[8] E. Fridman and U. Shaked. Delay-dependent stability and $H_{\infty}$ control: constant and time-varying delays. International Journal of Control 76(1):48-60, 2003.

[9] H. Gao, T. Chen, and J. Lam. A new delay system approach to network-based control. Automatica 44(1):39-52, 2008.

[10] K. Gu, V. Kharitonov, and J. Chen, Stability of Time-Delay Systems. Control Engineering Series. Birkhauser, Boston, 2003.

[11] I. Haidar, P. Mason, S.-I. Niculescu, M. Sigalotti, and A. Chaillet Further remarks on Markus-Yamabe instability for time-varying delay differential equations. In Proceedings of the 12th IFAC Workshop on Time Delay Systems, Ann Arbor, MI, 2015, pp. 33-38.

[12] A. Halanay. Differential Equations: Stability, Oscillations, Time Lags. Academic Press, New York, 1966.

[13] J. Hale. Ordinary Differential Equations. Robert E. Krieger Publishing Company, Malabar, FL, 1997.

[14] H. Khalil. Nonlinear Systems, Third Edition. Prentice Hall, Englewood Cliffs, NJ, 2002.

[15] V. Kharitonov and S.-I. Niculescu. On the stability of linear systems with uncertain delay. IEEE Transactions on Automatic Control 48(1):127-132, 2002.

[16] M. Krstic. Lyapunov stability of linear predictor feedback for time-varying input delay. IEEE Transactions on Automatic Control 55(2):554-559, 2010.

[17] K. Liu, V. Suplin, and E. Fridman. Stability of linear systems with general sawtooth delay. IMA Journal of Mathematical Control and Information 27(4):419-436, 2010.

[18] J. Louisell. New examples of quenching in delay differential equations having time-varying delay. In Proceedings of the 5th European Control Conference, Karlsruhe, Germany, 1999.

[19] L. Markus and H. Yamabe. Global stability criteria for differential systems. Osaka Mathematical Journal 12(2):305-317, 1960.

[20] F. Mazenc and M. Malisoff. Trajectory based approach for the stability analysis of nonlinear systems with time delays. IEEE Transactions on Automatic Control 60(6):1716-1721, 2015.

[21] F. Mazenc, M. Malisoff, and T. Dinh. Robustness of nonlinear systems with respect to delay and sampling of the controls. Automatica 49(6): 1925-1931, 2013.

[22] F. Mazenc, M. Malisoff, and S.-I. Niculescu. Reduction model approach for linear time-varying systems with delays. IEEE Transactions on Automatic Control 59(8):2068-2081, 2014.

[23] W. Michiels and S.-I. Niculescu. Stability and Stabilization of TimeDelay Systems. Advances in Design and Control. Society for Industrial and Applied Mathematics, Philadeplphia, PA, 2007.

[24] L. Mirkin. Some remarks on the use of time-varying delay to model sample-and-hold circuits. IEEE Transactions on Automatic Control 52(6): 1109-1112, 2007.

[25] B. Zhou. Truncated Predictor Feedback for Time-Delay Systems. Springer, London, UK, 2014. 